\title{
Retention of perampanel in adults with pharmacoresistant epilepsy at a single tertiary care center
}

Tim Wehner ${ }^{\mathrm{a}}$, Shahidul Mannan ${ }^{\mathrm{a}}$, Sanchit Turaga ${ }^{\mathrm{a}}$, Kirtana Vallabhaneni ${ }^{\mathrm{a}}$, Hao Meng Yip ${ }^{\mathrm{a}}$, Carys Wiggans ${ }^{\mathrm{b}}$, Rohit Shankar ${ }^{\mathrm{b}}$, John S Duncan ${ }^{\mathrm{a}}$, Josemir W Sander ${ }^{\mathrm{a}, \mathrm{c}}$

aNIHR UCL Hospitals Biomedical Research Centre, UCL Institute of Neurology, Queen Square WC1N 3BG, and Chalfont Centre for Epilepsy, Chalfont St Peter SL9 0RJ, UK

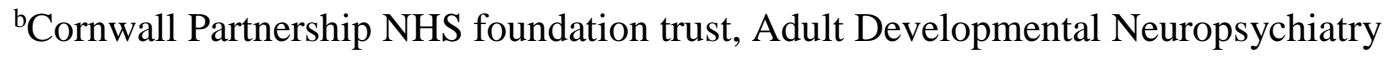
Chygovenck, Threemilestone Industrial Estate, Threemilestone Truro TR4 9LD UK ${ }^{c}$ Stichting Epilepsie Instellingen Nederland (SEIN), Achterweg 5 Heemstede 2103SW, The Netherlands

Email Addresses:

Tim Wehner $\quad \underline{\text { tim.wehner@gmail.com }}$

Shahidul Mannan shahidul.mannan@uclh.nhs.uk

Sanchit Turaga $\quad$ Sanchit.turaga.11@ucl.ac.uk

Kirtana Vallabhaneni kirtana.vallabhaneni.12@ucl.ac.uk

Hao Meng Yip hao.yip.12@ucl.ac.uk

Carys Wiggans carys.wiggans@nhs.net

Rohit Shankar_Ｒohit.shankar@nhs.net 
John S Duncan $\quad$ j.duncan@ucl.ac.uk

Josemir W Sander 1.sander@ucl.ac.uk

Corresponding author:

Dr Tim Wehner*

Department of Clinical Neurophysiology, Box 141

National Hospital for Neurology and Neurosurgery

Queen Square

London WC1N 3BG UK

Phone: $+44-20-34488619$

Fax: FAX

Email: tim.wehner@gmail.com

Current addree: Dr Tim Wehner Knappschaftskrankenhaus Bochum, Klinik für Neurologie, In der Schornau 23, 44892 Bochum, Germany

Abstract: 312 words

Manuscript: 2069 words excluding tables and references 


\section{Conflicts of interest}

The UCL Institute of Neurology has a long standing collaboration with Epilepsy Society which administers the Chalfont Centre. Eisai, the manufacturer of perampanel, supports activities at Epilepsy Society. Eisai had no role in the collection and analysis of the data or the drafting of the manuscript.

All authors read and understood the policy on conflicts of interests. SM, ST, KV, HMY and CW have no conflicts of interest to disclose. TW has received speaker fees for participation at educational activities organised by Eisai and Pfizer. RS has received institutional and research support and personal fees from UCB, Eisai, Janssen, Lilly, GSK, Servier, Astra Zeneca and Desitin outside the submitted work. JSD has a research collaboration with Medtronic. JWS has received research grants and honoraria from UCB, Eisai, Teva, Lundbeck and GSK which are involved in the manufacturing of AEDs. .

\section{Abstract}

Rationale: Observational data on antiepileptic drugs (AEDs) inform about their use in clinical practice. We describe our clinical experience with perampanel (PER) in a large UK tertiary epilepsy center.

Methods: Adults initiated on PER between October 2012 and March 2015 were followed until they discontinued PER or 10 September 2016. Data on epilepsy syndrome, duration, seizure types, concomitant and previous AED use, PER dosing, efficacy and side effects were recorded. Efficacy was categorized as temporary or ongoing (at last follow up) seizure freedom, $\geq 50 \%$ 
seizure reduction, or other benefit (e. g. No convulsions or daytime seizures). These categories were mutually exclusive except for people with temporary seizure freedom.

Results: 391 received a PER prescription, five of whom never took it. No follow up data were available for ten. $83 \%$ had focal epilepsy. People were prescribed PER in addition to 1-7 (Interquartile range [IQR] 2, 2, 3) AEDs and had previously used up to 18 (IQR 5, 7, 10) AEDs. Total exposure was 639 patient/years. Retention rates were $60.4 \%$ at one year, $48.3 \%$ at two years, and $42.7 \%$ at three years. $19(5 \%)$ people reported seizure free periods lasting at least six months. $A \geq 50 \%$ reduction in seizures lasting at least six month was reported by 76 people (20\%), and marked improvement for $\geq 6$ months was seen in $52(14 \%)$. Five (1\%) were taken off other AEDs and continued onPER monotherapy for $4-27$ months. Seizures aggravated in 57 (15\%). Somatic side effects were reported by 197 (52\%), mostly CNS. Mood changes, irritability or challenging behaviour were reported by 137 (36\%). PER was discontinued by $211(56 \%)$ due to adverse effects (39\%), inefficacy (26\%), or both (35\%). No idiosyncratic adverse events were seen. .

Conclusion: PER resulted in some benefit in $40 \%$ of those exposed. Adverse effects on mental health and on balance were common and should be discussed with people before initiating PER.

Highlights:

- Single center observation of 376 adults with epilepsy treated with perampanel

- Perampanel retention was $60 \%$ at one year, $48 \%$ at 2 years, $43 \%$ at 3 years

- $73 \%$ reported adverse effects, largely affecting the CNS (52\%) or mental health (36\%). 
Key words:, Cox regression analysi, antiepileptic drugs, drug resistant, treatment 


\section{Introduction ${ }^{1}$}

PER is a recently introduced AED which acts as a non-competitive antagonist at AMPA receptors in the brain, so reducing neuronal excitability.[1] PER was licensed as add-on treatment in focal epilepsy following results from three randomised controlled trials in people with pharmacoresistant epilepsy. [2][3][4]

Regulatory trials establish whether an AED is effective, but do not inform about its use in "reallife" population with epilepsy, many of whom might not meet the inclusion criteria for these trials for a number of reasons. We have evaluated the use of newly introduced AEDs over the past 20 years.[5][6][7][8][9][10] Here, we present a similar observation on the use of PER.

\footnotetext{
${ }^{1}$ Abbreviations:

AED - antiepileptic drug IQR - interquartile range

PER - perampanel

SD - standard deviation
}

SUDEP - sudden unexplained death in epilepsy 


\section{Patients and Methods}

Adults 17 years and older with epilepsy who received their first prescription for PER at the epilepsy specialist clinics at National Hospital for Neurology and Neurosurgery (Queen Square and Chalfont sites) between 1 October 2012 and 31 March 2015 were identified using the hospital's central pharmacy database and the departmental database. People started on PER elsewhere were excluded to avoid referral bias. Data regarding epilepsy syndrome, seizure types, age at onset of epilepsy, psychiatric comorbidity (if listed in records or inferred by use of antidepressant or antipsychotic medications), presence of learning disability, and use of current and previous AEDs, date of starting and stopping (where applicable) PER, maximum attained dose and maintenance dose were extracted from records. All started PER as add-on treatment. The starting dose was $2 \mathrm{mg}$ per day and was typically increased every two to four weeks by $2 \mathrm{mg}$ as tolerated. At our center, people with epilepsy are typically seen every six months, though they are encouraged to make phone contact in between if needed. All encounters with epilepsy care providers were analyzed to assess effect on seizure frequency and severity, adverse effects, and reasons to discontinue PER where applicable. Effect on seizures was categorized as seizure freedom for $>5$ times the average interval in between seizures during the previous year, a reduction in seizures by $50 \%$ or more, any other marked improvement in the assessment of either the individual or the physician, or worsening of seizures. Examples of marked improvement were cessation of convulsions, significant shortening of seizure or postictal confusion duration or reduction in seizure related falls or injuries. These categories were mutually exclusive except for periods of temporary seizure freedom (i. e. a person who reported initial seizure freedom of six months, followed by $>50 \%$ reduction in seizure freedom for 12 months, appeared in both categories). We only considered beneficial effects lasting six months or longer in the analysis to 
avoid the regression to mean phenomenon.[11] Seizure aggravation was noted if there was a significant increase in seizure frequency or seizure related morbidity. People were followed until they discontinued PER; data were censored on 10 September 2016 for those continuing on PER. Current AED use was assessed on the day of starting PER; i. e. changes in AEDs other than PER were not captured. Three people underwent temporal lobe resections after starting PER. Improvement following surgery was not attributed to PER. This exercise was approved as an audit, and no ethics approval was necessary.

Data were collected in a Microsoft Excel Spreadsheet. Statistical analysis was performed using SPSS 16 (SPSS Inc, Chicago, IL) using descriptive statistics, Fisher's exact test, Mann-Whitney tests, Kaplan-Meier Survival analysis, and Cox regression analysis as indicated in the manuscript. 


\section{Results}

\subsection{Baseline characteristics and dosing}

391 people received a prescription for PER between 22 October 2012 and 31 March 2015. For five, there was no subsequent evidence that they ever took the drug. No follow up data were available for ten people. The analysis thus includes 376 adults who took PER for a period of 4 1389 days (censored), a total of 639 patient-years exposure. Demographic variables are given in table 1. People were prescribed PER in addition to 1-7 (IQR 2, 2, 3) AEDs and had documented use of 1-18 (IQR 5, 7, 10) further AEDs. Table 2 lists AEDs used in addition to PER in at least $5 \%$ of people.

Tables 1, 2 and 3 about here

The maximum attained dose of PER was 2 - $16 \mathrm{mg}$ (IQR 4, 8, 10) $\mathrm{mg}$ in all people, and 1 - 14 (IQR 6, 8, 10) mg in those who continued the drug at last follow up (table 3). Men attained a higher maximum dose than women, and people on at least one enzyme-inducing AED (carbamazepine, phenytoin, phenobarbitone, primidone, topiramate) at the time of PER initiation attained a higher maximum dose of PER compared to those on no enzyme-inducing AEDs. Those who reported side effects attained lower maximum doses than those who did not.

\subsection{Benefits of perampanel}

19 people $(5 \%)$ reported periods of seizure freedom lasting 6 months or more. This benefit was temporary for up to 19 (median 9) months in ten, and ongoing at last follow up for up to 38 (18) 
months in nine. A reduction in seizure frequency by $>50 \%$ for 6 months or longer was seen in $76(20 \%)$, temporary for up to 36 (17) months in 31 and ongoing at last follow up for up to 42 (28) months in 45. A marked improvement for a minimum of 6 months was noted by 52 people (14\%), temporary for up to 33 (12) months in 12 and ongoing at last follow up for up to 41 (24) months in 40.57 people (15\%) reported seizure aggravation resulting in discontinuation of PER in most cases and significant reduction of the dose in the remaining. Five people discontinued other AEDs and converted to PER monotherapy. People who reported periods of seizure freedom lasting six months or more had failed fewer AEDs (mean 7.4, median 6, range 1-13) than those who did not report this benefit (mean 10.1, median 10, range 2-21, $\mathrm{p}=0.003$ ). There were no differences in the number of previously failed AEDs for the subgroups who reported seizure reduction by $50 \%$ or more for six months or longer, or those who reported "marked improvement" for six months or longer, versus those who did not.

\subsection{Adverse events on perampanel}

197 people (52\%) reported side effects, largely drowsiness, dizziness, and unsteadiness. A negative effect on mental health (worsening of mood, increased irritability, or challenging behavior was observed in 137 (36\%). One man required hospitalization for a psychotic episode shortly after starting PER. One man got arrested and another one cautioned for aggressive behavior. No idiosyncratic side effect was seen. There were two deaths in the cohort, one from myocardial infarction, one from complications of longstanding pulmonary hypertension. Neither case was attributed to PER. No cases of SUDEP were observed.

\subsection{Retention and Cox regression analysis}


Estimated retention of PER was $60.4 \%$ (95\% CI $55.4-65.3 \%)$ at one year, $48.3 \%(43.2$ $53.3 \%)$ at two years, and $42.7 \%(37.5-48 \%)$ at three years (figure). As of 10 September 2016, 211 people $(56 \%)$ have discontinued PER due to side effects $(82,39 \%)$, inefficacy or worsening of seizures $(55,26 \%)$, or both $(74,35 \%)$. Univariate Cox regression analysis (table 4$)$ suggests a significant $(\mathrm{p}<0.05)$ positive association with PER retention for concomitant use of pregabalin, maximum attained dose, periods of seizure freedom, $50 \%$ seizure reduction, or other significant improvement. Psychiatric side effects (most commonly irritability $(\mathrm{n}=81)$ and depression $(\mathrm{n}=41))$, and seizure aggravation were negatively associated with PER retention. Age at epilepsy onset, age at starting PER, epilepsy syndrome, presence of psychiatric comorbidity or learning disability, number of concomitant AEDs and number of failed AEDs did not affect retention.

We then performed a stepwise multivariate backwards regression analysis including all parameters that had been found to have an association (positive or negative) with PER retention at a level of $\mathrm{p}<0.2$ (table 5). Concomitant use of pregabalin and maximum attained dose remained positively associated with PER retention, as did periods of benefits as described above. Worsening of seizures, presence of psychiatric or somatic side effects and concomitant use of zonisamide were negatively associated with PER retention at a significance of $p<0.05$.

Figure, Table 4 and 5 about here

There were no differences in the number of people who reported somatic or psychiatric side effects when comparing the subgroups who were on concomitant pregabalin or zonisamide when starting PER with those who were not on these AEDs. There were also no differences in the 
continuing or maximum dose attained in those on concomitant pregabalin or zonisamide versus those not on these AEDs. 


\section{Discussion}

4.1. Comparison to regulatory trials and other retention reports

A temporary or ongoing benefit lasting at least six months was reported by nearly $40 \%$ of people following the introduction of PER. These results are comparable to the efficacy reported in the regulatory trials (studies 304-306) [2][3][4], though inferior to the results of an open label extension study reporting seizure reduction of up to $58 \%$ in people who took PER for two years.[12] This difference may be attributed to the fact that three quarters of people in our cohort had failed at least seven AEDs prior to commencing PER. Side effects in this cohort were similar to those reported previously in regulatory trials. No idiosyncratic side effects emerged. This is similar to previous reports. [13][14] No cases of SUDEP were observed in these 639 patients/years of exposure. The estimated incidence of SUDEP is $\sim 1$ in 200 people with refractory epilepsy per year.[15] We thus would have expected at least one case of SUDEP during this period but numbers are small.

Retention rates at one, two and three years in this cohort were similar to those observed in our historical cohort of people on lacosamide at one (60\% for PER vs $62 \%$ for lacosamide) and two years (48\% vs 45\%), and slightly better at three years (43\% vs 35\%).[9] While these two cohorts were similar in regards to their demographic characteristics, people in the PER cohort had failed one more AED (median) than those in the lacosamide cohort.

Prior observational studies on PER use have reported 12 months retention rates of $46-60.6 \%$ $[16][17][18][19][20]$, with broadly similar rates and types of side effects.

During enrolment, PER was licensed as add-on treatment for focal seizures only. The drug was used "off- label" in $17 \%$ of the cohort at the discretion of the prescribing physician. In June 
2015, the indication for PER was expanded to add-on in primary generalized tonic clonic seizures.

There were no differences in PER retention or attained doses of PER for people age 60 years or older compared to those younger than 60 years, in line with the findings of a post-hoc analysis of people age 65 and above in the PER regulatory trials.[21]

Adverse effects on mental health were reported by more than one third of people in line previous reports.[17][19] We did not systematically collect data with regards to specific side effects that lead to discontinuation. Psychiatric side effects judged to be severe in this cohort were observed in three. The possibility of these side effects should be discussed with individuals and PER should be used cautiously in people with a history of aggressive behavior.

PER retention was positively affected by concomitant use of pregabalin and negatively by zonisamide. There were no differences in the presence of somatic or psychiatric adverse effects, and no differences in the doses attained in those who took PER with or without combination with pregabalin or zonisamide. No pharmacodynamic or pharmacokinetic interaction between pregabalin and PER or zonisamide and PER have been yet described.[1][22] The observed effects of concomitant use of certain AEDs on PER retention might be a statistical artefact, the potential beneficial effect of pregabalin was the factor with the strongest modifiable effect in both univariate and multivariate Cox regression analysis. We therefore believe that this potential association deserves further investigation.

From a practical standpoint, our data suggest that a substantial minority of people with pharmacoresistant epilepsy experience some form of benefit from PER, despite having failed many AEDs. Further observations are warranted to determine whether PER might be more 
beneficial in people who have failed fewer AEDs. Adverse effects on mental health are the most relevant in clinical practice. These should be thoroughly discussed with individuals and actively explored after starting PER.

\subsection{Limitations}

There are a number of limitations: PER effects were not prospectively assessed but retrospectively collected and may thus be subject to recall and reporting bias. We relied on data that were not systematically captured during routine clinical encounters and documented in medical records only. The documented use of previous AEDs therefore may have underestimated the actual use, in particular in those who had apparently failed only few AEDs prior to PER. These effects were, however, judged to be small and thus unlikely to affect the analysis on retention. Seizure counts have been shown to be inaccurate [23], and no systematic use of seizure diaries was ensured. Beneficial effects in few individuals may have been falsely attributed to PER in the presence of another intervention (e. g. further subsequent drug change in rare cases), though we did not attribute benefits that appeared after resective epilepsy surgery. 
Acknowledgements:

We thank Dr Kanjana Unnwongse, Prasad Neurological Institute, Bangkok, Thailand, for help with the statistical analysis.

We are grateful to all clinicians whose patients have been included. We thank the pharmacists Evelyn Frank and Eisha Gosrani for assistance in identifying patients from UCLH trust's prescription databases.

These data were presented in part at the American Epilepsy Society Meeting in 2016. Raw data of this observation have been shared with a Pan-European effort (lead: E Trinka, Salzburg, Austria) to provide pooled observational data.

Raw data of some people in this audit were also shared with the Epilepsy and Intellectual disability register.

Funding: This work was supported by the National Institute for Health Research University College London Hospitals Biomedical Research Centre. 


\begin{tabular}{|c|c|c|}
\hline Gender (female) & 219 & $58 \%$ \\
\hline Age when starting PER (years) & $32,42,50(\mathrm{IQR})$ & $17-82$ (range) \\
\hline Age at epilepsy onset (years) & $5,12,19$ (IQR) & first year of life -79 \\
\hline Psychiatric history & 101 & $27 \%$ \\
\hline Learning disability & 68 & $18 \%$ \\
\hline Focal epilepsy & 314 & $83 \%$ \\
\hline Symptomatic & 193 & $51 \%$ \\
\hline Cryptogenic & 121 & $32 \%$ \\
\hline Generalised epilepsy & 58 & $16 \%$ \\
\hline Symptomatic & 26 & $7 \%$ \\
\hline Cryptogenic & 25 & $7 \%$ \\
\hline Idiopathic & 7 & $2 \%$ \\
\hline Unclassified & 4 & $1 \%$ \\
\hline $\begin{array}{l}\text { Number of AEDs previously tried } \\
\text { (excluding current AEDs) with } \\
\text { evidence in electronic medical records }\end{array}$ & 7 (median) & 0-18 (range) \\
\hline $\begin{array}{l}\text { Number of AEDs previously tried } \\
\text { (including current AEDs) }\end{array}$ & 10 (median) & $1-21$ (range) \\
\hline \multicolumn{3}{|l|}{ Number of concomitant AEDs } \\
\hline One & 52 & $14 \%$ \\
\hline Two & 137 & $36 \%$ \\
\hline three & 121 & $32 \%$ \\
\hline Four or more & 66 & $18 \%$ \\
\hline
\end{tabular}


Table 1: Demographic characteristics of people in the cohort. 


\begin{tabular}{|l|c|c|}
\hline Antiepileptic drug & $\mathrm{N}$ & $\%$ \\
\hline Carbamazepine & 143 & 38 \\
\hline Levetiracetam & 133 & 35 \\
\hline Clobazam & 117 & 31 \\
\hline Lamotrigine & 85 & 23 \\
\hline Lacosamide & 75 & 20 \\
\hline Zonisamide & 65 & 17 \\
\hline Sodium Valproate & 64 & 17 \\
\hline Oxcarbazepine & 62 & 17 \\
\hline Phenytoin & 42 & 11 \\
\hline Topiramate & 41 & 11 \\
\hline Pregabalin & 32 & 9 \\
\hline Clonazepam & 22 & 6 \\
\hline Phenobarbitone & 19 & 5 \\
\hline
\end{tabular}

Table 2: Antiepileptic drugs used at time of initiating perampanel in at least 5\% of the cohort

\begin{tabular}{|c|c|c|c|c|}
\hline \multirow[b]{2}{*}{ All people } & \multicolumn{2}{|c|}{$\begin{array}{l}\text { Maximum dose (mg) } \\
\text { (number of people) }\end{array}$} & \multicolumn{2}{|c|}{$\begin{array}{l}\text { Continuing dose (mg) } \\
\text { (number of people) }\end{array}$} \\
\hline & $7.3 \pm 3.1(376)$ & p-value ${ }^{2}$ & $\begin{array}{l}7.5 \pm 3.3 \\
(165)\end{array}$ & p-value ${ }^{2}$ \\
\hline Men & $7.9 \pm 3.0(157)$ & & $7.8 \pm 3.1(75)$ & \\
\hline Women & $6.9 \pm 3.1(219)$ & 0.001 & $7.3 \pm 3.4(90)$ & 0.411 \\
\hline People on $\geq 1$ enzyme inducing AED ${ }^{1}$ & $7.6 \pm 3.1(214)$ & & $8.1 \pm 3.1(101)$ & \\
\hline People on no enzyme inducing AED ${ }^{1}$ & $6.9 \pm 2.9(162)$ & 0.029 & $6.7 \pm 3.3(64)$ & 0.007 \\
\hline People $<60$ years old ${ }^{1}$ & $7.4 \pm 3.1(342)$ & & $7.7 \pm 3.3(151)$ & \\
\hline People $\geq 60$ years old ${ }^{1}$ & $6.3 \pm 2.8(34)$ & 0.083 & $6.3 \pm 3.1(14)$ & 0.143 \\
\hline People who reported adverse effects & $7.0 \pm 2.9(276)$ & & $7.0 \pm 3.2(105)$ & \\
\hline People who did not report adverse effects & $8.1 \pm 3.3(100)$ & 0.003 & $8.4 \pm 3.3(60)$ & 0.011 \\
\hline People with somatic adverse effects & $6.9 \pm 2.9(197)$ & & $7.1 \pm 3.2(82)$ & \\
\hline People without somatic adverse effects & $7.7 \pm 3.2(179)$ & 0.032 & $8.0 \pm 3.4(83)$ & 0.093 \\
\hline People with psychiatric adverse effects & $6.9 \pm 2.7(137)$ & & $6.4 \pm 3.1(45)$ & \\
\hline $\begin{array}{l}\text { People without psychiatric adverse } \\
\text { effects }\end{array}$ & $7.5 \pm 3.2(239)$ & 0.055 & $7.9 \pm 3.3(120)$ & 0.008 \\
\hline
\end{tabular}

SD, standard deviation

${ }^{1}$ at time of starting perampanel

${ }^{2}$ two-tailed Mann Whitney test, uncorrected for multiple comparisons

Table 3: Maximum and continuing doses of PER attained in subgroups 
Factors positively associated with perampanel retention

\begin{tabular}{|l|c|c|c|}
\hline & Odds Ratio $^{1}$ & $95.0 \% \mathrm{CI}^{1}$ & p-value \\
\hline Concomitant use of pregabalin $(\mathrm{n}=32)$ & 0.39 & $0.20-0.76$ & 0.006 \\
\hline Concomitant use of oxcarbazepine $(\mathrm{n}=62)$ & 0.75 & $0.51-1.10$ & 0.144 \\
\hline Presence of simple partial seizures $(\mathrm{n}=179)$ & 0.77 & $0.59-1.01$ & 0.057 \\
\hline Maximum dose attained & 0.80 & $0.76-0.84$ & $<0.001$ \\
\hline Male gender $(\mathrm{n}=157)$ & 0.80 & $0.61-1.06$ & 0.115 \\
\hline Duration of seizure freedom $(\mathrm{n}=19)$ & 0.88 & $0.80-0.96$ & 0.005 \\
\hline Number of concomitant AEDs used & 0.89 & $0.78-1.01$ & 0.078 \\
\hline Duration of marked improvement $(\mathrm{n}=52)$ & 0.89 & $0.85-0.94$ & $<0.001$ \\
\hline $\begin{array}{l}\text { Duration of }>50 \% \text { improvement of seizure } \\
\text { frequency }(\mathrm{n}=76)\end{array}$ & 0.93 & $0.91-0.96$ & $<0.001$ \\
\hline
\end{tabular}

Factors negatively associated with perampanel retention

\begin{tabular}{|l|c|c|c|}
\hline & Odds ratio $^{1}$ & $95 \% \mathrm{CI}^{1}$ & p-value \\
\hline Worsening of seizures $(\mathrm{n}=56)$ & 2.75 & $1.99-3.79$ & $<0.001$ \\
\hline Concomitant use of gabapentin $(\mathrm{n}=8)$ & 2.10 & $0.99-4.47$ & 0.054 \\
\hline Presence of psychiatric side effects $(\mathrm{n}=137)$ & 1.55 & $1.18-2.04$ & 0.002 \\
\hline Previous use of retigabine $(\mathrm{n}=96)$ & 1.43 & $1.06-1.91$ & 0.018 \\
\hline Previous use of oxcarbazepine $(\mathrm{n}=104)$ & 1.36 & $1.02-1.82$ & 0.039 \\
\hline Concomitant use of zonisamide $(\mathrm{n}=65)$ & 1.30 & $0.93-1.82$ & 0.126 \\
\hline Previous use of lacosamide $(\mathrm{n}=201)$ & 1.26 & $0.96-1.66$ & 0.095 \\
\hline Presence of somatic side effects $(\mathrm{n}=197)$ & 1.20 & $0.91-1.57$ & 0.195 \\
\hline Number of previous AEDs used & 1.02 & $0.99-1.06$ & 0.183 \\
\hline
\end{tabular}

${ }^{1}$ Odds ratio and $95 \%$ confidence interval of the probability of discontinuing perampanel.

Table 4: Results of univariate Cox regression analysis. Only factors with a p-value of $<0.2$ are listed. 
Factors positively associated with perampanel retention

\begin{tabular}{|l|c|c|c|}
\hline & Odds Ratio $^{1}$ & $95 \% \mathrm{CI}^{1}$ & p-value \\
\hline Concomitant use of pregabalin $(\mathrm{n}=32)$ & 0.49 & $0.25-0.96$ & 0.038 \\
\hline Maximum dose attained & 0.78 & $0.73-0.82$ & $<0.001$ \\
\hline Duration of marked improvement $(\mathrm{n}=52)$ & 0.88 & $0.84-0.93$ & $<0.001$ \\
\hline Duration of seizure freedom $(\mathrm{n}=19)$ & 0.89 & $0.83-0.96$ & 0.003 \\
\hline $\begin{array}{l}\text { Duration of }>50 \% \text { improvement of seizure } \\
\text { frequency }(\mathrm{n}=76)\end{array}$ & 0.92 & $0.90-0.95$ & $<0.001$ \\
\hline
\end{tabular}

Factors negatively associated with perampanel retention

\begin{tabular}{|l|c|c|c|}
\hline & Odds Ratio $^{1}$ & $95 \%$ CI $^{1}$ & p-value \\
\hline Worsening of seizures $(\mathrm{n}=56)$ & 2.33 & $1.65-3.30$ & $<0.001$ \\
\hline Presence of psychiatric side effects $(\mathrm{n}=137)$ & 1.78 & $1.34-2.38$ & $<0.001$ \\
\hline Concomitant use of zonisamide $(\mathrm{n}=65)$ & 1.53 & $1.09-2.16$ & 0.015 \\
\hline Previous use of lacosamide $(\mathrm{n}=201)$ & 1.49 & $1.12-1.98$ & 0.006 \\
\hline Presence of somatic side effects $(\mathrm{n}=197)$ & 1.49 & $1.13-1.96$ & 0.005 \\
\hline Previous use of oxcarbazepine $(\mathrm{n}=104)$ & 1.36 & $1.01-1.84$ & 0.043 \\
\hline
\end{tabular}

${ }^{1}$ Odds ratio and $95 \%$ confidence interval of the probability of discontinuing perampanel.

Table 5: Results of multivariate Cox regression analysis. All factors listed in table 4 were included in a stepwise backword logistic regression analysis until only factors with a p-value $<0.05$ remained.

Figure: retention of perampanel 
References

[1] Patsalos PN. The clinical pharmacology profile of the new antiepileptic drug perampanel: A novel noncompetitive AMPA receptor antagonist. Epilepsia 2015;56:12-27.

[2] French JA, Krauss GL, Biton V, Squillacote D, Yang H, Laurenza A, et al. Adjunctive perampanel for refractory partial-onset seizures: Randomized phase III study 304. Neurology 2012;79:589-96.

[3] French JA, Krauss GL, Steinhoff BJ, Squillacote D, Yang H, Kumar D, et al. Evaluation of adjunctive perampanel in patients with refractory partial-onset seizures: Results of randomized global phase III study 305. Epilepsia 2013;54:117-25.

[4] Krauss GL, Serratosa JM, Villanueva V, Endziniene M, Hong Z, French J, et al. Randomized phase III study 306 . Adjunctive perampanel for refractory partial-onset seizures. Neurology 2012;78:1408-15.

[5] Lhatoo SD, Wong ICK, Polizzi G, Sander JW. Long-term retention rates of lamotrigine, gabapentin, and topiramate in chronic epilepsy. Epilepsia 2000;41:1592-6.

[6] Depondt C. The long term retention of levetiracetam in a large cohort of patients with epilepsy. J Neurol Neurosurg Psychiatry 2006;77:101-3.

[7] Yuen AWC, Singh R, Bell GS, Bhattacharjee A, Neligan A, Heaney DC, et al. The longterm retention of pregabalin in a large cohort of patients with epilepsy at a tertiary referral centre. Epilepsy Res 2009;87:120-3.

[8] Catarino CB, Bartolini E, Bell GS, Yuen AWC, Duncan JS, Sander JW. The long-term retention of zonisamide in a large cohort of people with epilepsy at a tertiary referral centre. Epilepsy Res 2011;96:39-44.

[9] Novy J, Bartolini E, Bell GS, Duncan JS, Sander JW. Long-term retention of lacosamide in a large cohort of people with medically refractory epilepsy: a single centre evaluation. Epilepsy Res 2013;106:250-6.

[10] Wehner T, Chinnasami S, Novy J, Bell GS, Duncan JS, Sander JW. Long term retention of retigabine in a cohort of people with drug resistant epilepsy. Seizure 2014;23:878-81.

[11] Spilker BA, Segreti A. Validation of the phenomenon of regression of seizure frequency in epilepsy. Epilepsia 1984;25:443-9.

[12] Krauss GL, Perucca E, Ben-Menachem E, Kwan P, Shih JJ, Clément JF, et al. Long-term safety of perampanel and seizure outcomes in refractory partial-onset seizures and secondarily generalized seizures: Results from phase III extension study 307 . Epilepsia 2014;55:1058-68.

[13] Laurenza A, Yang H, Williams B, Zhou S, Ferry J. Absence of Liver Toxicity in Perampanel-Treated Subjects: Pooled results from partial seizure phase III perampanel clinical studies. Epilepsy Res 2015;113:76-85.

[14] Yang H, Laurenza A, Williams B, Patten A, Hussein Z, Ferry J. Lack of effect of perampanel on QT interval duration: Results from a thorough QT analysis and pooled partial seizure Phase III clinical trials. Epilepsy Res 2015;114:122-30.

[15] Devinsky O, Hesdorffer DC, Thurman DJ, Lhatoo S, Richerson G. Sudden unexpected death in epilepsy: epidemiology, mechanisms, and prevention. Lancet Neurol 2016;15:1075-88.

[16] Brodie MJ, Stephen LJ. Prospective audit with adjunctive perampanel: Preliminary observations in focal epilepsy. Epilepsy Behav 2016;54:100-3.

[17] Coyle H, Clough P, Cooper P, Mohanraj R. Clinical experience with perampanel: Focus on 
psychiatric adverse effects. Epilepsy Behav 2014;41:193-6.

[18] Juhl S, Rubboli G. Perampanel as add-on treatment in refractory focal epilepsy. The Dianalund experience. Acta Neurol Scand 2016:374-7.

[19] Maurousset A, Limousin N, Praline J, Biberon J, Corcia P, De Toffol B. Adjunctive perampanel in refractory epilepsy: Experience at tertiary epilepsy care center in Tours. Epilepsy Behav 2016;61:237-41.

[20] Villanueva V, Garcés M, López-González FJ, Rodriguez-Osorio X, Toledo M, Salas-Puig J, et al. Safety, efficacy and outcome-related factors of perampanel over 12 months in a real-world setting: The FYDATA study. Epilepsy Res 2016;126:201-10.

[21] Leppik IE, Wechsler RT, Williams B, Yang H, Zhou S, Laurenza A. Efficacy and safety of perampanel in the subgroup of elderly patients included in the phase III epilepsy clinical trials. Epilepsy Res 2015;110:216-20.

[22] Patsalos PN. Drug Interactions with the Newer Antiepileptic Drugs (AEDs) - Part 1 : Pharmacokinetic and Pharmacodynamic Interactions Between AEDs. Clin Pharmakokinet 2013:927-66.

[23] Hoppe, C; Poepel, A; Elger C. Epilepsy: accuracy of patient seizure counts. Arch Neurol 2007:1595-9. 